\title{
Zero-temperature properties of the quantum dimer model on the triangular lattice
}

\author{
Arnaud Ralko, ${ }^{1}$ Michel Ferrero, ${ }^{2}$ Federico Becca, ${ }^{2}$ Dmitri Ivanov, ${ }^{1}$ and Frédéric Mila ${ }^{1}$ \\ ${ }^{1}$ Institut de Théorie des Phénomènes Physiques, Ecole Polytechnique Fédérale de Lausanne (EPFL), CH-1015 Lausanne, Switzerland \\ ${ }^{2}$ INFM-Democritos, National Simulation Centre and International School for Advanced Studies (SISSA), Via Beirut 2-4, \\ I-34014 Trieste, Italy \\ (Received 11 February 2005; published 23 June 2005)
}

\begin{abstract}
Using exact diagonalizations and Green's function Monte Carlo simulations, we have studied the zerotemperature properties of the quantum dimer model on the triangular lattice on clusters with up to 588 sites. A detailed comparison of the properties in different topological sectors as a function of the cluster size and for different cluster shapes has allowed us to identify different phases, to show explicitly the presence of topological degeneracy in a phase close to the Rokhsar-Kivelson point, and to understand finite-size effects inside this phase. The nature of the various phases has been further investigated by calculating dimer-dimer correlation functions. The present results confirm and complement the phase diagram proposed by Moessner and Sondhi on the basis of finite-temperature simulations [Phys. Rev. Lett. 86, 1881 (2001)].
\end{abstract}

DOI: 10.1103/PhysRevB.71.224109

PACS number(s): 75.10.Jm, 05.50.+q, 05.30.-d

\section{INTRODUCTION}

The investigation of spin-liquid phases is currently a very active field of research, partly_but not only-because of their possible connection to the superconductivity observed in several cuprates. The definition of a "spin liquid" is itself a matter of debate. Following the work of Shastry and Sutherland on a two-dimensional model whose exact ground state is a product of dimer singlets, ${ }^{1}$ the word is sometimes used to designate phases in which the spin-spin correlation function decays exponentially fast with distance at zero temperature. However, such phases often exhibit other types of long-range order, such as dimer order, which manifest themselves as nondecaying correlation functions involving more than two spins. ${ }^{2}$ In that respect, the word liquid is not appropriate, and it should arguably be reserved for systems in which all correlation functions decay exponentially fast at large distance. This discussion would be quite academic if the only characteristic of such liquids was the absence of any kind of order, but following the pioneering work of Wen, ${ }^{3}$ it is well admitted by now that such liquids can exhibit another property known as topological order: In the thermodynamic limit, the ground state (when defined on a topologically nontrivial domain) exhibits a degeneracy not related to any symmetry and referred to as topological degeneracy. These degenerate ground states live in topological sectors which cannot be connected by any local operator.

The realization of such phases in quantum spin models is still preliminary though. The best candidates are frustrated magnets for which quantum fluctuations are known to destroy magnetic long-range order, but their ground-state properties are very difficult to access, and when definite conclusions are reached, it is usually because the presence of some kind of long-range order (dimer, plaquette, etc.) can be established. ${ }^{4}$ The main difficulty is in a sense technical: A good diagnosis would require us to study large enough clusters, but this is not possible since quantum Monte Carlo simulations of frustrated antiferromagnets are plagued with a very severe minus sign problem.

In that respect, effective models such as the quantum dimer model (QDM) are extremely interesting. Although their relationship to actual Heisenberg antiferromagnets is not a simple issue, ${ }^{5}$ they describe resonance processes typical of strongly fluctuating frustrated quantum magnets while retaining the possibility to be analyzed by standard techniques such as quantum Monte Carlo. This possibility was first exploited by Moessner and Sondhi, ${ }^{6}$ who developed a finitetemperature Monte Carlo algorithm to study the QDM on a triangular lattice defined by the Hamiltonian

$$
\begin{aligned}
& H=v \sum(|\Xi\rangle\langle\Xi|+| \boldsymbol{U}\rangle\langle\boldsymbol{U}|) \\
& -t \sum(|\Xi\rangle\langle\mu|+|\langle\rangle\langle\Xi|),
\end{aligned}
$$

where the sum runs over all plaquettes including the three possible orientations. The kinetic term controlled by the amplitude $t$ changes the dimer covering of every flippable plaquette, i.e., of every plaquette containing two dimers facing each other, while the potential term controlled by the interaction $v$ describes a repulsion $(v>0)$ or an attraction $(v<0)$ between dimers facing each other. Since a positive $v$ favors configurations without flippable plaquettes while a negative $v$ favors configurations with the largest possible number of flippable plaquettes, the so-called maximally flippable plaquette configurations (MFPC), one might expect a phase transition between two phases as a function of $v / t$. The actual situation is far richer, though. As shown by Moessner and Sondhi, who calculated the temperature dependence of the structure factor, there are four different phases (see Fig. 1): (i) A staggered phase for $v / t>1$, in which the ground-

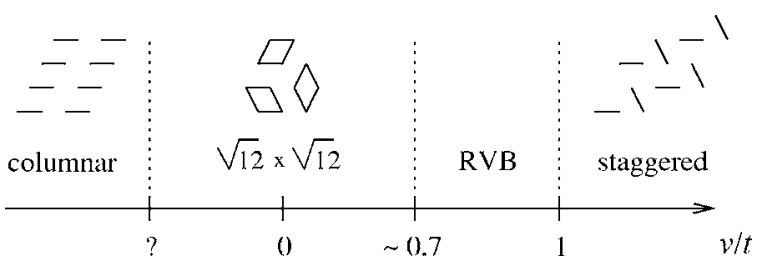

FIG. 1. Phase diagram of the QDM on the triangular lattice as a function of $v / t$ after Ref. 6. 
state manifold consists of all nonflippable configurations; (ii) a columnar ordered phase for $v / t$ sufficiently negative; (iii) an ordered phase adjacent to it called the $\sqrt{12} \times \sqrt{12}$ phase where the structure factor develops a low-temperature peak at an intermediate value of the wave vector and which probably consists of resonating plaquettes which make a 12 -site unit-cell pattern; (iv) a liquid phase with a featureless and temperature-independent structure factor. This last phase has been interpreted as a short-range resonating valence bond (RVB) phase in which all correlations decay exponentially, even at low temperature. ${ }^{7}$ It is separated from the staggered phase by a special point, the Rokhsar-Kivelson (RK) point $(v / t=1)$. At this point, the ground-state manifold contains all staggered configurations plus another configuration of zero energy, the sum of all possible configurations. In that particular state, the dimer-dimer correlation function has been shown to decay exponentially with distance at zero temperature. $^{6}$

While the arguments put forward by Moessner and Sondhi in favor of this phase diagram are quite convincing, this proposal calls for further investigation for several reasons. First of all, this zero-temperature phase diagram was inferred from finite-temperature results, and a direct analysis of ground-state properties would be welcome. Besides, the location of the phase boundaries is to a large extent unknown, as well as the nature of the quantum phase transitions between the different phases. Finally, and more importantly, a direct investigation of the RVB phase would help to understand its physical properties. In particular, this phase is expected to be liquid in the strong sense of the word, and, as such, to have topological degeneracy, but this could not be checked by the finite-temperature calculation of Moessner and Sondhi. In fact, this property, which lies at the root of Ioffe et al.'s proposal for q-bits, ${ }^{8}$ has never been directly observed. As noticed by Ioffe and collaborators on the basis of exact diagonalizations of small clusters, the finite-size effects are still huge for the small cluster sizes accessible with that technique, and no conclusion regarding the thermodynamic limit could be reached.

In this paper, we address all these issues, and answer most of them, by a careful investigation of the zero-temperature properties of the model essentially based on the implementation of a Green's function Monte Carlo (GFMC) algorithm. The huge finite-size effects are shown to be a natural consequence of the subtle interplay between the cluster geometry, the order parameter of the underlying phase (if any), and the topological sector. This analysis turns the finite-size effects into a very powerful tool to investigate the ground-state properties of the model as a function of $v / t$. In particular, we have obtained strong evidence in favor of topological degeneracy close to the RK point, and we have been able to locate the transition between the two ordered phases with a reasonable accuracy.

The paper is organized as follows. In Sec. II, we present the minimal technical background necessary to understand the results presented in the following sections. To keep this technical section as small as possible, some details have been relegated into Appendixes. We then discuss the various phases and the transitions between them in Sec. III, and finally we conclude in Sec. IV.

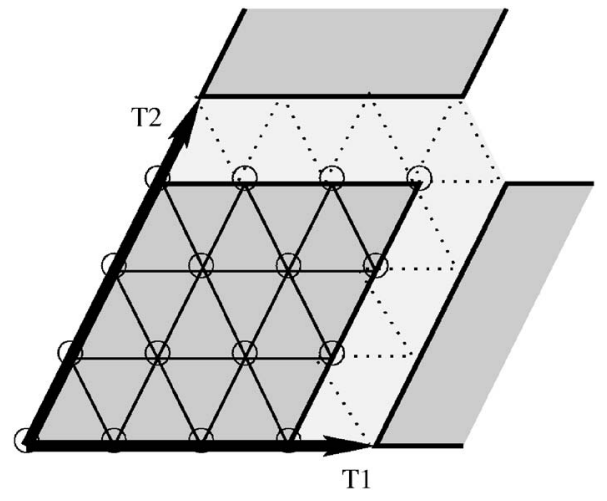

FIG. 2. Example of a type-A cluster with 16 sites.

\section{TECHNICAL BACKGROUND}

\section{A. Topological sectors}

On the triangular lattice, the QDM has conserved quantities defining topological sectors (TS). ${ }^{6,9,10}$ On finite clusters, these conserved quantities are defined by the parity of the number of dimers intersecting a given line which satisfies two properties: (i) It is closed, or it ends at the boundaries of the cluster; (ii) it does not divide the cluster into two disconnected pieces. The first condition ensures that the parity is conserved when applying the Hamiltonian, while the second condition guarantees the possibility to construct configurations with both parities. On a cylinder, the only choice is a line going from one end to the other, while on a torus, one can choose any closed loop that goes around one of the axes of the torus. Since the sectors defined by two lines that can be deformed into each other continuously are the same, one ends up with two sectors for a cylinder and four sectors for a torus. In the following, we will work exclusively with clusters defined on a torus.

\section{B. Finite clusters}

On the triangular lattice, it is possible to construct two types of clusters which keep all the symmetries of the infinite lattice. ${ }^{11}$ In terms of the basis vectors $\mathbf{u}_{1}$ and $\mathbf{u}_{2}$ [with $\mathbf{u}_{1}$ $=(1,0)$ and $\left.\mathbf{u}_{2}=(1 / 2, \sqrt{3} / 2)\right]$, they are defined by two vectors,

$$
\begin{gathered}
\mathbf{T}_{1}=l \mathbf{u}_{1}+m \mathbf{u}_{2}, \\
\mathbf{T}_{2}=-m \mathbf{u}_{1}+(l+m) \mathbf{u}_{2}
\end{gathered}
$$

with $l$ or $m=0$ for type-A clusters (see Fig. 2) and $l=m$ for type-B clusters (see Fig. 3). The clusters have the geometry of tori obtained by identifying sites of the infinite lattice modulo the vectors $\mathbf{T}_{1}$ and $\mathbf{T}_{2}$.

The number of sites $N$ of such clusters is given by the simple formula $N=l^{2}+l m+m^{2}$, leading to $N=l^{2}$ sites for type-A clusters and $N=3 \times l^{2}$ sites for type-B clusters. Since the number of sites must be even to accommodate dimers, we consider only clusters with $l$ even.

\section{Topological sectors, order, and cluster size}

The four topological sectors that we denote with $(0,0)$, $(0,1),(1,0)$, and $(1,1)$ can be defined by considering loops 


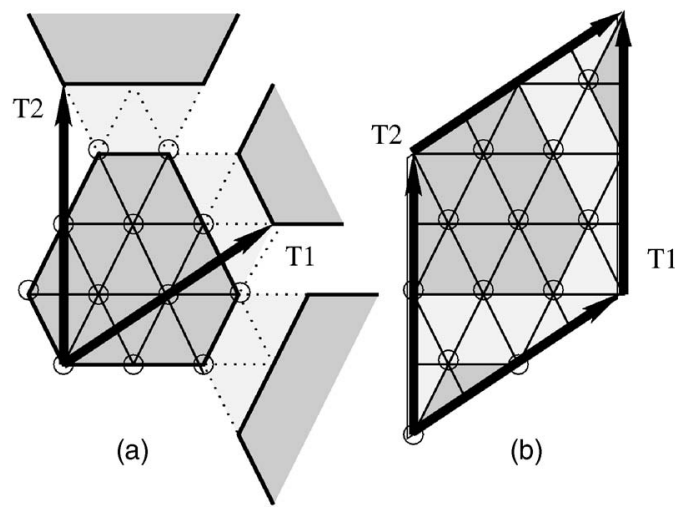

FIG. 3. Example of type-B clusters with 12 sites showing the two different choices of unit cell.

along $\mathbf{u}_{1}$ and $\mathbf{u}_{2}$, where the first (second) number refers to the loop along $\mathbf{u}_{1}\left(\mathbf{u}_{2}\right)$, and 0 (1) stands for even (odd). Remarkably, even on a finite cluster, three of the four sectors are always equivalent: This property is a consequence of the correspondence between sectors under rotations (see Appendix A) and applies equally to type-A and type-B clusters, and, therefore, the three sectors have the same spectrum. However, which sectors are degenerate depends on the size of the cluster, according to the rule of Fig. 4.

If the system is in a completely liquid phase with no longrange order at all, all topological sectors are expected to be equivalent up to finite-size effects, and each topological sector should contain one replica of the ground state. However, if some dimer order develops, this is no longer true, and the ground state will only be found in some sectors. The rule depends on the cluster size and on the type of order. The columnar order can only appear in the $(0,0)$ sector, and, according to the rule of Fig. 4 , in the $(0,1)$ and $(1,0)$ sector when $l / 2$ is odd. On the other hand, the $\sqrt{12} \times \sqrt{12}$ phase is not frustrated in clusters with a number of sites multiple of 12 , i.e., all type-B clusters and type-A clusters with $l$ multiple of 3 . Further, it is only compatible with the $(0,0)$ sector for $l / 2$ even and with the $(1,1)$ sector when $l / 2$ is odd. This rule is again equally applicable to both type-A and type-B clusters. The above properties are summarized in Table I. They will prove helpful to identify the phases and their boundaries since we only have access to ground-state properties in different topological sectors.

\section{Numerical methods}

Since the size of the Hilbert space grows exponentially, exact diagonalizations could only be performed for one cluster of type B (12 sites) and two clusters of type A (16 and 36

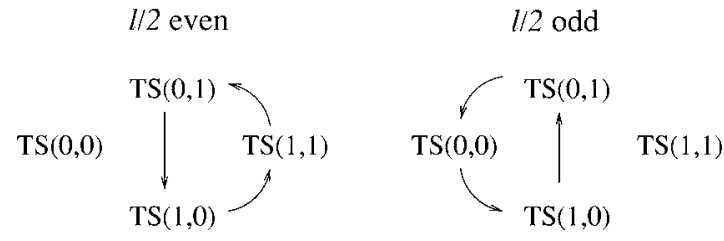

FIG. 4. Degeneracy of the TS as a function of the parity of $l / 2$. Three out of four TS are degenerate since they contain the same configurations rotated by an angle $\pi / 3$.
TABLE I. Topological sectors in which the various states and phases discussed in the text can be found. The symbol * means that the size of the cluster must be a multiple of 12 to be compatible with the $\sqrt{12} \times \sqrt{12}$ phase.

\begin{tabular}{ccccc}
\hline \hline \multicolumn{1}{c}{$l / 2$} & MFPC & Columnar & $\sqrt{12} \times \sqrt{12}$ & RVB liquid \\
\hline even & all & $\operatorname{TS}(0,0)$ & $\operatorname{TS}(0,0)^{*}$ & all \\
& $\operatorname{TS}(0,0)$ & $\operatorname{TS}(0,0)$ & & \\
\multirow{2}{*}{ odd } & $\operatorname{TS}(0,1)$ & $\operatorname{TS}(0,1)$ & $\operatorname{TS}(1,1)^{*}$ & all \\
& $\mathrm{TS}(1,0)$ & $\operatorname{TS}(1,0)$ & & \\
\hline \hline
\end{tabular}

sites). A few typical results are listed in Appendix B. However, on the basis of these results only, not much could be said regarding the issues of topological degeneracy in the thermodynamic limit and of the phase transitions.

Fortunately, since all nondiagonal matrix elements are nonpositive, the zero-temperature GFMC method could be implemented. ${ }^{12}$ In particular, we make use of the algorithm with a fixed number of walkers described in Ref. 13. The algorithm is ergodic at sufficiently large $v / t$, except for the staggered configurations, which anyway play no role in the parameter range $v / t<1$ in which we are interested. However, the large energy barriers present for negative values of $v / t$, typically $v / t<-1$, make it difficult to browse the configuration space efficiently. In particular, for very negative $v / t$, the probability to sample states around the MFPC starting from a generic one becomes exponentially small, introducing serious ergodicity problems in the algorithm (Appen$\operatorname{dix} \mathrm{C})$.

Results have been obtained with this algorithm for clusters with up to 588 sites. While no guiding function was required very close to the RK point, a guiding function favoring clusters according to the number of flippable plaquettes had to be implemented to keep a good statistics when decreasing $v / t$.

\section{THE PHASE DIAGRAM}

We now turn to the presentation of the results we have obtained starting from the RK point and decreasing toward the most negative values of $v / t$ affordable by GFMC. As mentioned previously, the analysis is based on a comparison of the ground-state energy in different topological sectors. Since the sectors $(0,1)$ and $(1,0)$ are always degenerate with $(0,0)$ or $(1,1)$, depending on the parity of $l / 2$, a comparison of these last two sectors is sufficient. In the following, we will denote by $E_{(0,0)}\left(E_{(1,1)}\right)$ the total ground-state energy in sector $(0,0)[(1,1)]$ and by $\Delta E$ the difference between them: $\Delta E=E_{(1,1)}-E_{(0,0)}$, which we call the topological gap.

\section{A. The RVB phase}

In this section we present our numerical results close to the RK point, where a disordered spin-liquid phase is expected. We therefore look for evidence of topological degeneracy in the thermodynamic limit by a size-scaling study. Our analysis has shown that finite-size effects are very dif- 


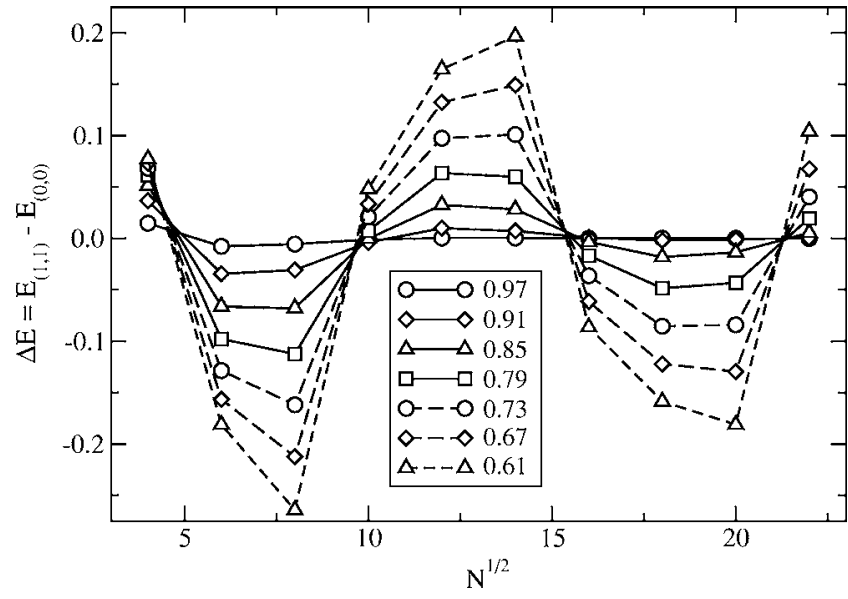

FIG. 5. Topological gap for type-A clusters as a function of the linear size $l=N^{1 / 2}$ of the cluster. The error bars are smaller than the symbol size.

ferent for clusters of type A and B, and we prefer to discuss them separately.

\section{Type-A clusters}

We have calculated the topological gap for type-A clusters with up to the $22 \times 22$ cluster and the results for the topological gap are shown in Fig. 5. The finite-size effects are very strong, and using only the exact diagonalization data, which extend up to $l=6$, it would be hopeless to draw any conclusion about the thermodynamic limit. Instead, with the sizes reachable within GFMC, a clear tendency appears: The topological gap seems to decrease with the cluster size with an oscillatory behavior. It has been shown in Ref. 10 that the same behavior must be observed in the correlation function of auxiliary fermions introduced in the Pfaffian method at the RK point. This correlation function behaves along $\mathbf{u}_{1}$ (or $\mathbf{u}_{2}$ ) as ${ }^{9,10}$

$$
G(R) \propto R^{-1 / 2} \exp (-R / \xi), \quad R \rightarrow \infty,
$$

where $\xi$ has both a real and an imaginary part given by $\xi^{-1}$ $\simeq 0.83+i 0.12 \pi .{ }^{14}$ Accordingly, we have tried to fit our results for the topological gap with an oscillatory function defined as

$$
\Delta \epsilon(l)=\frac{\text { const }}{l^{1 / 2}} e^{-\operatorname{Re}\left[\xi^{-1}\right] l} \cos \left(\operatorname{Im}\left[\xi^{-1}\right] l+\phi\right),
$$

where $\Delta \epsilon=\Delta E / N$ is the energy difference per site. Figure 6 displays these fits for three different ratios of $v / t$ near the RK point. As expected, the fit is very accurate for $v / t$ close to the RK point, but becomes worse as $v / t$ decreases.

The real and imaginary parts of the inverse correlation length $\xi^{-1}$ extracted from these fits are reported in Fig. 7. The continuous lines correspond to the values of $v / t$ for which the fit with Eq. (4) was sufficiently reliable. As $v / t \rightarrow 1$, these values connect smoothly to the analytic results obtained by Ioselevich and collaborators at the RK point.

Because $\operatorname{Re}\left[\xi^{-1}\right]$ remains finite away from the RK point, we can conclude that the topological gap vanishes exponen-

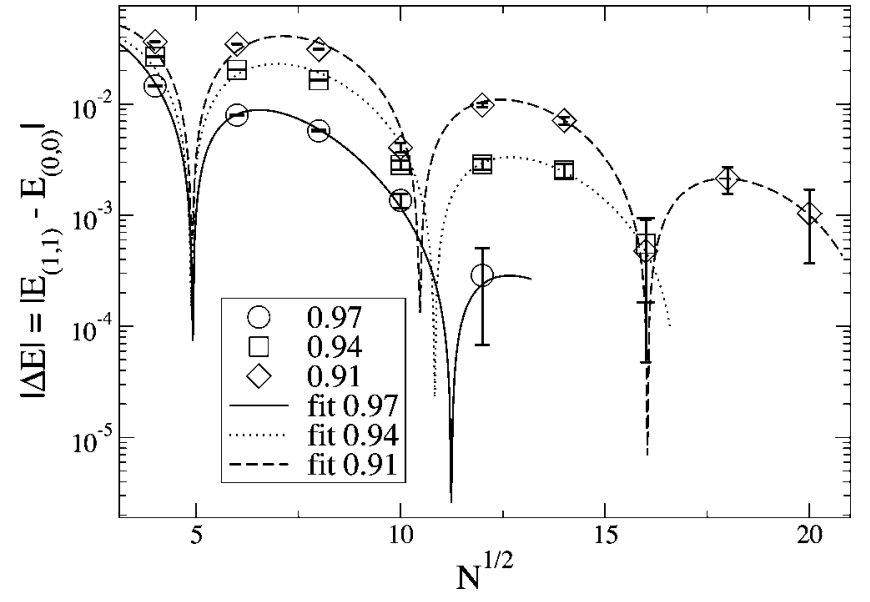

FIG. 6. Oscillatory fits of the topological gap per site near the RK point for type-A clusters. The plot is in a log-linear scale.

tially in the thermodynamic limit in a finite parameter range close to the RK point, as expected in the RVB phase. For $v / t<0.85, \operatorname{Im}\left[\xi^{-1}\right]$ saturates at the value corresponding to the $\sqrt{12} \times \sqrt{12}$ crystal phase and we shall comment on this fact in Sec. III B. The nontrivial behavior of the topological gap for different sizes of the cluster suggests to look at clusters of different geometry. Therefore, we continue our analysis with type-B clusters.

\section{Type-B clusters}

Now we consider the type-B clusters of size $N=3 \times l^{2}$ with $l$ up to 14 . The finite-size effects of the topological gap for this kind of cluster are much simpler than for type-A clusters. Indeed, $\Delta E$ changes sign according to $(-1)^{l / 2}$, and its absolute value smoothly decreases with the cluster size without any oscillations. Following Ref. 10, we may relate the topological gap in type-B clusters in the close vicinity of the RK point to the fermionic Green's function in the direc-

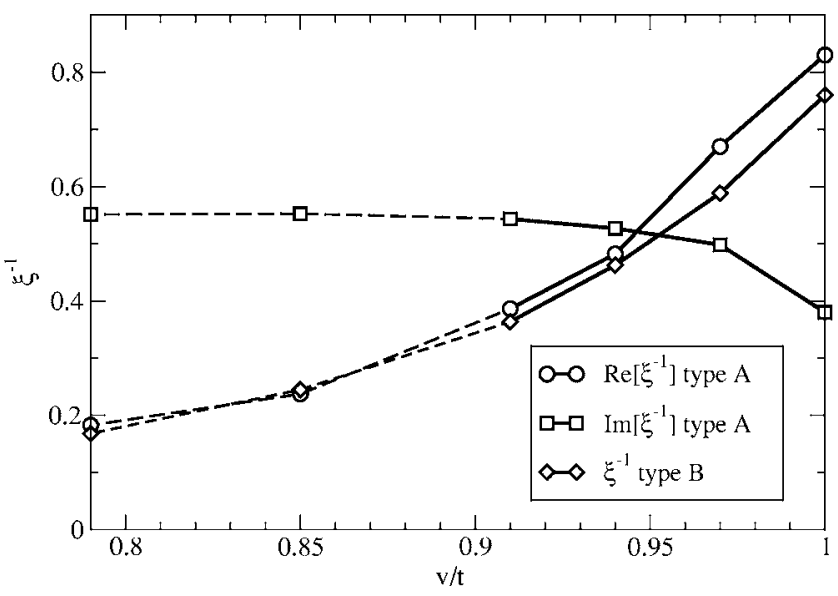

FIG. 7. Real and imaginary part of the inverse correlation length $\xi^{-1}$ as a function of $v / t$. At the RK point, the values quoted are the analytical result of Ref. 10. For type-A clusters, both real and imaginary parts of $\xi^{-1}$ are shown. The continuous lines connect the point for which the fit has a satisfactory accuracy (the error bars are of the order of the symbol size). 


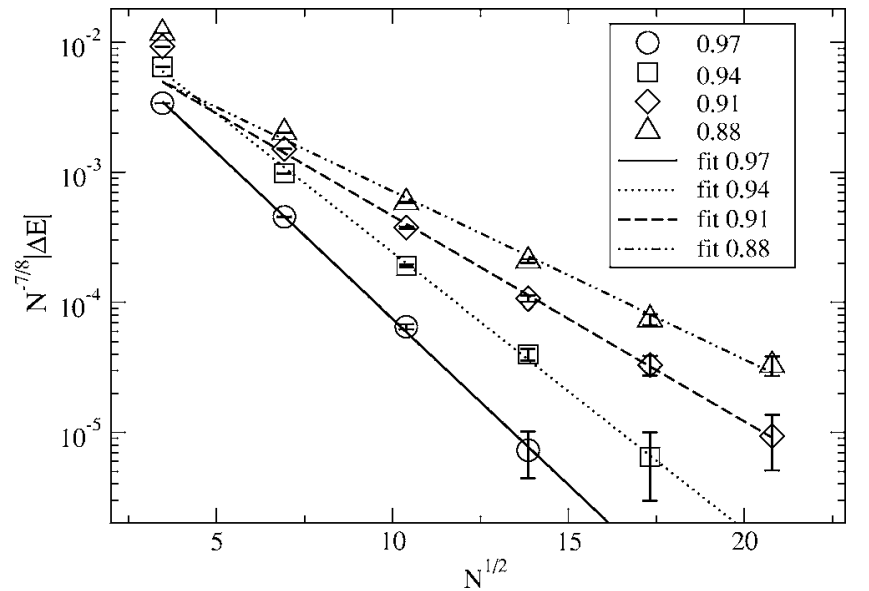

FIG. 8. Fits of the topological gap per site near the RK point for type-B clusters. We choose to multiply the total energy by the prefactor $N^{-7 / 8}$ to fit with a pure exponential function [see Eq. (6)].

tion $\mathbf{u}_{1}+\mathbf{u}_{2}$ ("B direction" in the terminology of Ref. 10). Because of the special symmetry properties along this direction, the corresponding correlation length is purely real at the RK point and its value is $\xi^{-1} \simeq 0.76$. An accurate calculation of the fermionic Green's function at the RK point along the lines of Ref. 9 gives

$$
G(R) \propto R^{-1 / 4} \exp (-R / \xi), \quad R \rightarrow \infty .
$$

Thus in the vicinity of the RK point we may fit the topological gap with ${ }^{15}$

$$
\Delta \epsilon(l)=\frac{\text { const }(-1)^{l / 2}}{l^{1 / 4}} e^{-\sqrt{3} l / \xi},
$$

where the correlation length $\xi$ is real $(\sqrt{3} l$ is the length of the vectors $\mathbf{T}_{1}$ and $\mathbf{T}_{2}$ in type-B clusters). Indeed, even away from the RK point, the "B direction" conserves its special symmetry, and we expect to see no incommensurate oscillations in $\Delta \epsilon(l)$. Our numerical calculations confirm this behavior, and as for type-A clusters, the fits are good close to $v / t=1$ if one only takes into account the sizes much larger than the correlation length (see Fig. 8). This exponential decay confirms the presence of topological degeneracy close to the RK point.

Remarkably, the $(-1)^{l / 2}$ oscillations in $\Delta \epsilon(l)$ connect smoothly the RVB phase to the $\sqrt{12} \times \sqrt{12}$ crystal phase, where the ground state must alternate between the $(0,0)$ and $(1,1)$ topological sectors. This indicates that short-range $\sqrt{12} \times \sqrt{12}$ correlations are probably already present in the ground state of the RVB phase.

\section{Correlation function}

To demonstrate the absence of long-range correlations in the vicinity of the RK point, we have also calculated the dimer-dimer correlation function $\langle D(x) D(0)\rangle$, where $D(x)$ is the dimer operator that is equal to 0 if there is no dimer on bond $x$ and to 1 if there is one. In the RVB liquid phase, we expect all dimer orientations to be equally probable far enough from the reference dimer $D(0)$. Since the average

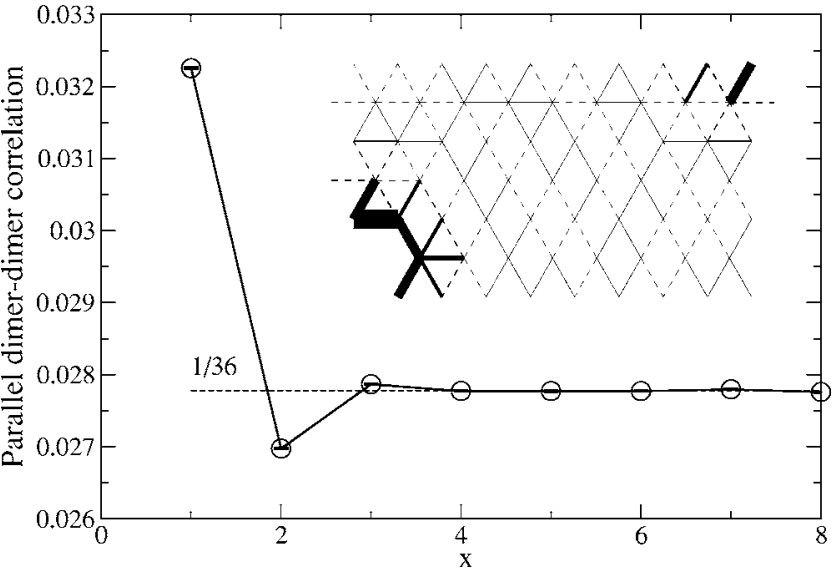

FIG. 9. Correlation function of two parallel dimers as a function of their separation $x$ along $\mathbf{u}_{1}$ for $v / t=0.97$. Inset: Dimer-dimer correlation function on the 108 -site cluster for $v / t=0.97$ in the topological sector $(1,1)$. Dashed (solid) links correspond to probabilities smaller (larger) than $1 / 36$, while the thickness of the links is proportional to the absolute value of the distance to $1 / 36$. The reference dimer is in the lower left corner.

dimer concentration is $1 / 6,\langle D(x) D(0)\rangle$ must tend to $1 / 36$ as $x \rightarrow \infty$. This liquid behavior of the dimer-dimer correlations has been confirmed at the RK point by Moessner and Sondhi, ${ }^{6,9}$ who have proven that, at $v / t=1,\langle D(x) D(0)\rangle$ tends to $1 / 36$ exponentially with increasing distance $x$.

Not surprisingly, we find the same behavior for $v / t$ close to 1, which lends further support to the existence of an RVB phase away from the RK point. An example of short-range dimer correlations at $v / t=0.97$ is shown in Fig. 9.

\section{B. The transition between the RVB and the intermediate $(\sqrt{12} \times \sqrt{12})$ phases}

Upon decreasing $v / t$, we expect a phase transition from the short-range RVB phase to an ordered phase with dimer or plaquette order. According to Moessner and Sondhi, this should occur somewhere between $v / t=0$ and $v / t=1$, presumably around $v / t=0.7$, and the phase on the other side of the transition should be the ordered $\sqrt{12} \times \sqrt{12}$ phase (see Fig. 10 for a schematic picture of the order or Refs. 6 and 16 for a more detailed discussion).
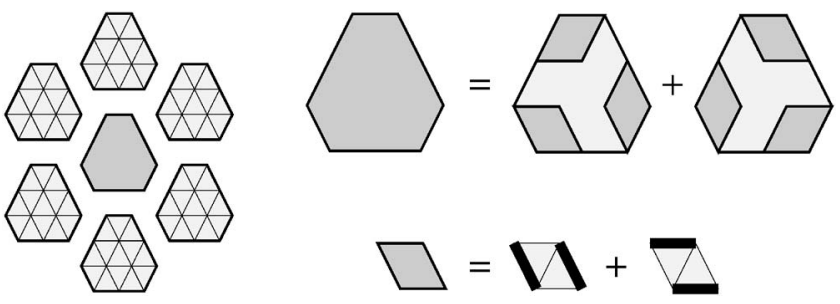

FIG. 10. A simplified scheme of the $\sqrt{12} \times \sqrt{12}$ phase. The unit cell (shaded hexagon) consists of 12 sites and contains three resonating rhombi plaquettes. This construction neglects correlations and resonances between different plaquettes of the same unit cell and between different unit cells. 


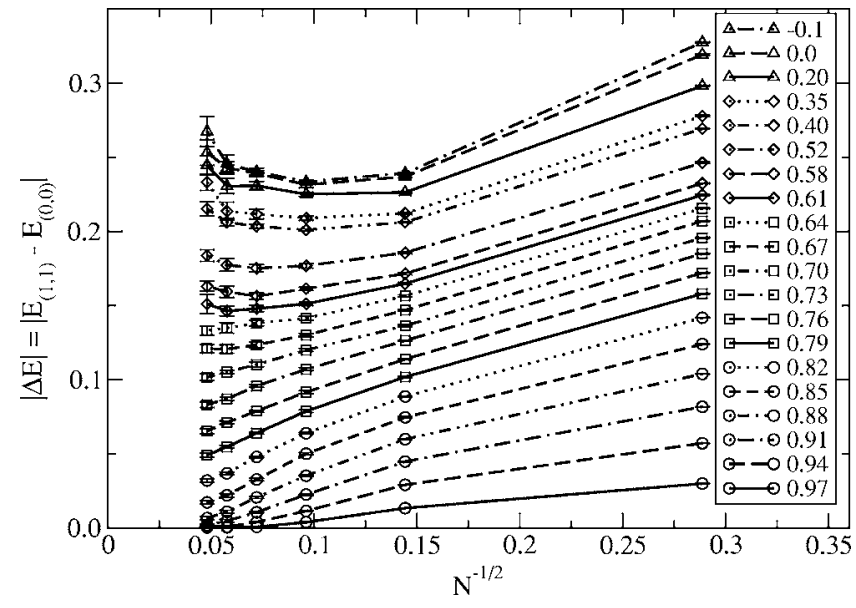

FIG. 11. Absolute value of the topological gap for type-B clusters as a function of $1 / \sqrt{N}$ for several values of $v / t$. There is a clear change of behavior between $v / t=1$ and $v / t=0$. Lines are guides for the eye.

From the results obtained on type-A clusters, one may suspect that the phase transition takes place around $v / t$ $=0.85$. Indeed, around this value $\operatorname{Im}\left[\xi^{-1}\right]$ saturates at a value close to $\pi / 6$, and at the same time the proposed fit (4) becomes inaccurate. Note that $\pi / 6$ is exactly the value one would expect for the periodicity of the topological gap in type-A clusters. Indeed, the period of the $\sqrt{12} \times \sqrt{12}$ crystal in the directions of $\mathbf{u}_{1}$ and $\mathbf{u}_{2}$ is six lattice spacings, and, therefore, the periodicity of the topological gap is 12 lattice spacings, since the ground-state topological sector changes as $l$ changes by 6 . The saturation value of $\operatorname{Im}\left[\xi^{-1}\right]$ reported in Fig. 7 is somewhat larger than $\pi / 6$, which is related to the inapplicability of the fitting formula (4) for small clusters. As one can measure directly from the last half-period of oscillations in Fig. 5, at $v / t \leqslant 0.85$ the correct value of the oscillation wave vector $\operatorname{Im}\left[\xi^{-1}\right]$ in fact equals $\pi / 6$ to a very good precision, in perfect agreement with $\sqrt{12} \times \sqrt{12}$ ordering.

Another estimate of the phase-transition point comes from the analysis of type-B clusters. The number of sites in these clusters is always a multiple of 12 ; therefore they can accommodate a $\sqrt{12} \times \sqrt{12}$ crystal without defects. As we have already mentioned, the sign oscillations of the topological gap $\Delta E=(-1)^{l / 2}|\Delta E|$ agree both with the RVB liquid and with the $\sqrt{12} \times \sqrt{12}$ crystal phase, and it is only the size dependence of $|\Delta E|$ that may indicate the phase transition.

We have plotted in Fig. 11 the absolute value of the topological gap as a function of $1 / \sqrt{N}$ for several values of $v / t$ ranging from 1 to negative values. There is a clear change of behavior for large clusters. For $v / t$ close to 1 , the topological gap monotonously decreases with the size, as discussed earlier, while for $v / t$ close to 0 , the topological gap seems to diverge with the size. This is actually what we expect in the $\sqrt{12} \times \sqrt{12}$ phase. Indeed, since this order can only appear in one topological sector (see Sec. II C), considering the wrong topological sector will require a one-dimensional defect, with an energy cost proportional to $\sqrt{N}$. We could not reach a large enough cluster to actually check this scaling form, but the data are consistent with it. The precise location of the transition is also difficult to pin down, but the change of
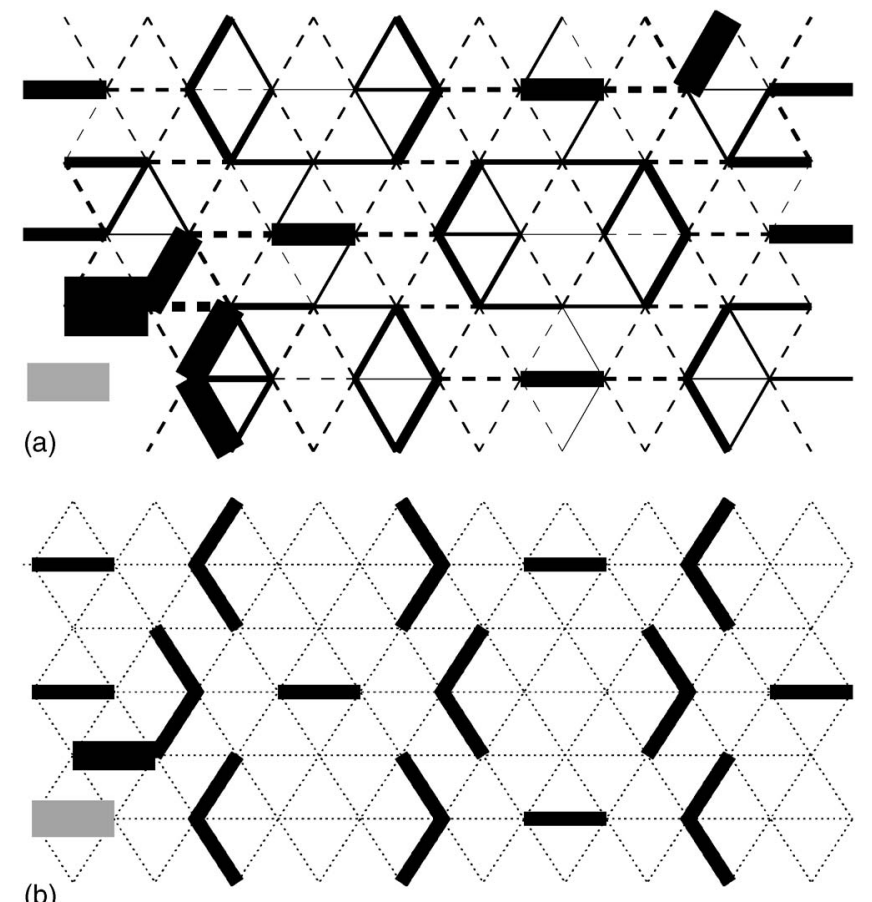

FIG. 12. Upper panel: ground-state dimer-dimer correlations of the 108 -site cluster for $v / t=0$ in the topological sector $(1,1)$. The same plotting scheme as in the inset of Fig. 9. Lower panel: the schematic plot of dimer-dimer correlations in the classical state of Fig. 10. Thick lines represent peaks of the correlation function higher than $50 \%$ above the average value $1 / 36$. The gray dimer in the bottom left corner represents the reference link.

curvature for the large sizes occurs around $v / t=0.7$, which may be taken as a candidate value for the transition point.

To summarize, from the analysis of topological gaps in type-A and type-B clusters, the phase-transition point between the RVB and the $\sqrt{12} \times \sqrt{12}$ phases may be estimated between $v / t \sim 0.7$ and $v / t \sim 0.85$. However, our results do not shed much light on the nature of the phase transition [see, e.g., a possible transition scenario with $\mathrm{O}(4)$ symmetry in Ref. 16]. We may only remark that the correlation lengths reported in Fig. 7 admit the possibility of a second-order phase transition: the correlation lengths for type-A and type-B clusters merge together quickly as $v / t$ decreases from the RK point (the correlation length should become isotropic and divergent at the second-order transition point). Such a transition would also explain our difficulties in locating the exact transition value of $v / t$ from simulations on small clusters.

\section{The intermediate $(\sqrt{12} \times \sqrt{12})$ phase}

In addition to the analysis of the topological gaps in type-A and type-B clusters, we may obtain more direct evidence of the existence of the $\sqrt{12} \times \sqrt{12}$ phase from the dimer-dimer correlation functions. Upon decreasing $v / t$ from the RK point, the long-distance correlations gradually increase. The resulting pattern for 108 sites and $v / t=0$ is shown in Fig. 12. 


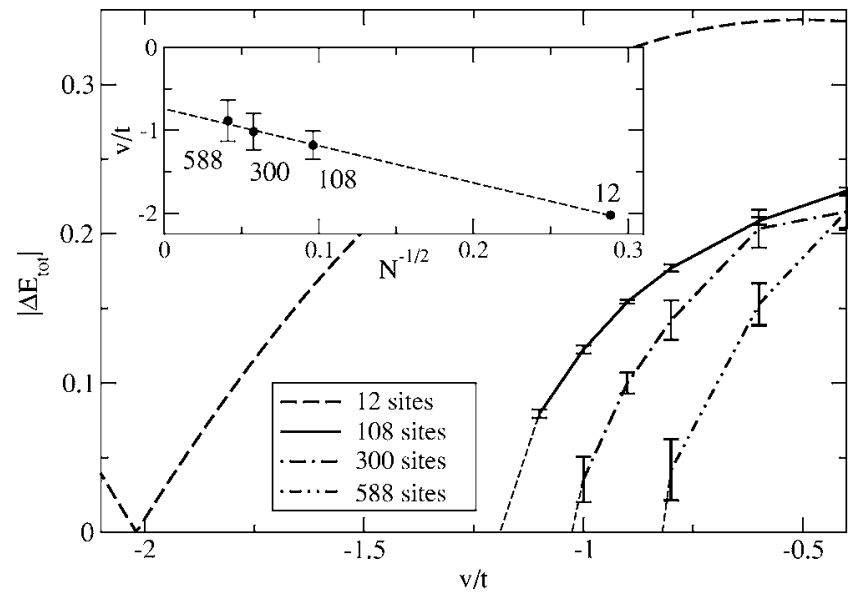

FIG. 13. Absolute value of the topological gap as a function of $v / t$ for type-B clusters with $12,108,300$, and 588 sites. The dashed lines are extrapolations to locate the level crossing between the $(0,0)$ and $(1,1)$ topological sectors. Inset: Linear extrapolation of the level crossing points.

We can identify the structure of these correlations as belonging to the intermediate $\sqrt{12} \times \sqrt{12}$ phase. For this purpose, we simulate the dimer-dimer correlations in a classical state corresponding to the schematic representation of the intermediate phase in Fig. 10. Namely, we assume that all dimer configurations within a fixed $\sqrt{12} \times \sqrt{12}$ crystal of hexagons come with equal probability. The resulting pattern of increased dimer-dimer correlations is presented in the lower panel of Fig. 12. Even though this construction is oversimplified, we expect that it correctly predicts the main features of the correlation function. A direct comparison with the upper panel of Fig. 12 indicates a $\sqrt{12} \times \sqrt{12}$ correlation pattern at $v / t=0$.

\section{The transition between the intermediate and the columnar phases}

According to Moessner and Sondhi, a phase transition to the columnar phase is expected when $v / t$ becomes sufficiently negative, although they were not able to locate it precisely. If we look at appropriate clusters, this transition should correspond to a change of ground-state topological sector. Indeed, while in the columnar phase the ground state always appears in the $(0,0)$ sector [and, for $l / 2$ odd, also in $(0,1)$ and $(1,0)]$, in the $\sqrt{12} \times \sqrt{12}$ phase, it appears in the $(0,0)$ sector if $l / 2$ is even and in the $(1,1)$ if $l / 2$ is odd (see Sec. II C). Therefore, if we look at a cluster multiple of 12 with $l / 2$ odd, the transition from the $\sqrt{12} \times \sqrt{12}$ to the columnar phase should correspond to a change of ground-state topological sector from $(1,1)$ to $(0,0)$. In other words, the topological gap is expected to vanish at that point. To avoid mixing the results of clusters with different geometries, we have concentrated on available type-B clusters with appropriate geometries, namely clusters with 12, 108, 300, and 588 sites. The results are reported in Fig. 13. For 12 sites, they are obtained by exact diagonalizations, and the level crossing occurs at $v / t=-2.022$. For the other sizes, they have been obtained with GFMC. Because of ergodicity problems,

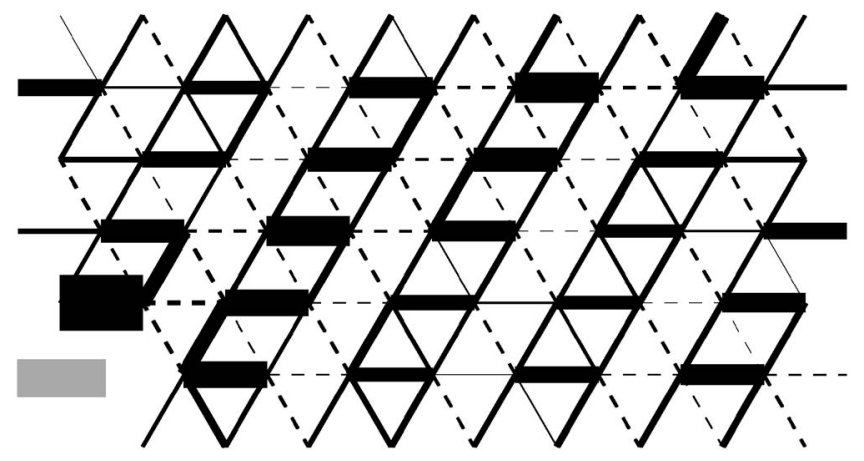

FIG. 14. Dimer-dimer correlation for the 108-site cluster at $v / t=-0.8$ in the topological sector $(0,0)$. A clear columnar pattern appears. The same plotting scheme as in the inset of Fig. 9.

appearing at negative $v / t$ (see Appendix C), we could not reach the level crossing, but we could get close enough to it to allow a meaningful extrapolation of the results. The results obtained are reported as a function of the inverse linear size $1 / \sqrt{N}$ in Fig. 13. It seems reasonable to approximate this size dependence by a linear function, which leads to an estimate $v / t=-0.75 \pm 0.25$ for the transition point between the $\sqrt{12}$ $\times \sqrt{12}$ phase and the columnar phase.

\section{E. The columnar phase}

Finally, although there is little doubt concerning the nature of the phase for large and negative $v / t$, we have tried to calculate the dimer-dimer correlation function in this phase as well for completeness. As mentioned before, there is an ergodicity problem, and we cannot reach that phase with GFMC. However, for clusters for which this transition appears as a level crossing between topological clusters, we can expect to get an idea of the correlations taking place in this phase by calculating them for a value of $v / t$ as close as possible to the transition and in the topological sector compatible with the columnar phase. We have thus calculated the dimer-dimer correlation function for the 108-site cluster for $v / t=-0.8$ in the topological sector $(0,0)$. The results are plotted in Fig. 14. A clear columnar pattern appears, in contrast to the correlations in the intermediate phase (see Fig. 12). Note that one columnar configuration has been selected from the others. This is due to the fact that the rotational symmetry is broken when one considers a single topological sector.

\section{CONCLUSIONS}

In this paper, we have performed a thorough analysis of the ground-state properties of the QDM on the triangular lattice with exact diagonalizations and GFMC. Results with respect to previous investigations could be obtained thanks to the combination of two factors: (i) The large sizes reachable with GFMC (up to 588 sites); (ii) the precise identification of the interplay between the cluster geometry, the topological sector, and the nature of the ground state.

The most important result of this paper is to provide direct numerical evidence that the four topological sectors on a torus are indeed degenerate in the thermodynamic limit in a 

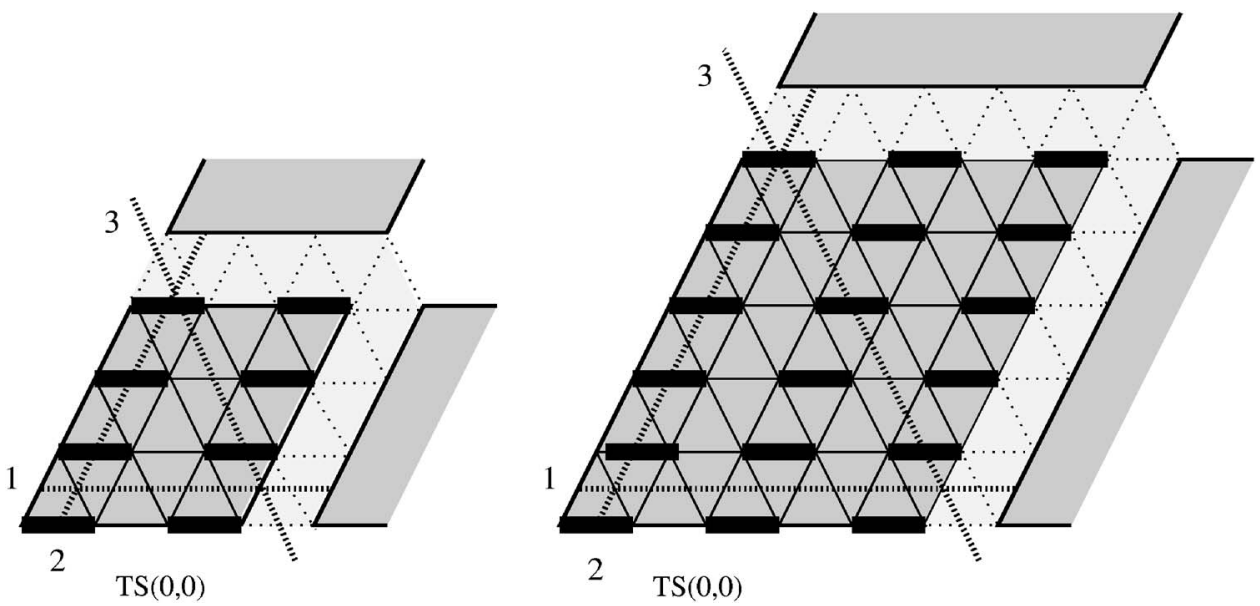

FIG. 15. Three cutlines labeled by 1,2 , and 3 on a type A cluster with (a) 16 sites ( $l / 2$ even) and (b) 36 sites (l/2 odd).

finite range below the RK point, thus confirming the identification of this phase as an RVB phase by Moessner and Sondhi. The finite-size effects toward this unique groundstate energy as revealed by the behavior of large clusters agree, in the vicinity of the RK point, with the analytic perturbative expressions of Ioselevich and collaborators. ${ }^{10}$ Furthermore, fitting with their perturbative formula allowed a meaningful extraction of the correlation length also away from $v / t=1$.

The other phases of the phase diagram of Moessner and Sondhi, namely the $\sqrt{12} \times \sqrt{12}$ and the columnar phases, have also been confirmed by an investigation of the dimerdimer correlation function, and a careful analysis of the crossing between two topological sectors for certain clusters

TABLE II. Total ground-state energy obtained with exact diagonalizations for the topological sectors $(0,0)$ and $(1,1)$ and for different sizes.

\begin{tabular}{ccccc}
\hline \hline$v / t$ & TS & 12 sites & 16 sites & 36 sites \\
\hline 0.8 & 00 & -0.69505 & -1.02863 & -2.10806 \\
& 11 & -0.84755 & -0.96885 & -2.20081 \\
0.6 & 00 & -1.48237 & -2.10582 & -4.36730 \\
& 11 & -1.70960 & -2.02804 & -4.55215 \\
0.4 & 00 & -2.31590 & -3.21999 & -6.74713 \\
& 11 & -2.58514 & -3.13239 & -6.98904 \\
0.2 & 00 & -3.17522 & -4.36297 & -9.21881 \\
& 11 & -3.47308 & -4.26664 & -9.48840 \\
0.0 & 00 & -4.05317 & -5.52971 & -11.76017 \\
& 11 & -4.37228 & -5.42488 & -12.03779 \\
-0.2 & 00 & -4.94745 & -6.71735 & -14.35767 \\
& 11 & -5.28161 & -6.60465 & -14.62961 \\
-0.4 & 00 & -5.85765 & -7.92454 & -17.00373 \\
& 11 & -6.20000 & -7.80513 & -17.25894 \\
-0.6 & 00 & -6.78415 & -9.15091 & -19.69534 \\
& 11 & -7.12645 & -9.02644 & -19.92244 \\
-0.8 & 00 & -7.72760 & -10.39677 & -22.43356 \\
& 11 & -8.06006 & -10.26927 & -22.61782 \\
\hline \hline
\end{tabular}

has allowed us to conclude that the phase transition takes place at $v / t=-0.75 \pm 0.25$.

The transition between the RVB phase and the $\sqrt{12}$ $\times \sqrt{12}$ phase is more difficult to pin down, but the behavior of the topological gap for large clusters suggests that it takes place between $v / t=0.7$ and $v / t=0.85$.

The main issue to be resolved is the nature of the phase transition between the RVB phase and the $\sqrt{12} \times \sqrt{12}$ phase. The correlation length increases away from the RK point, but

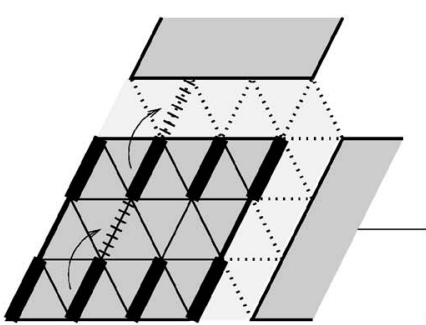

$\operatorname{TS}(0,0)$

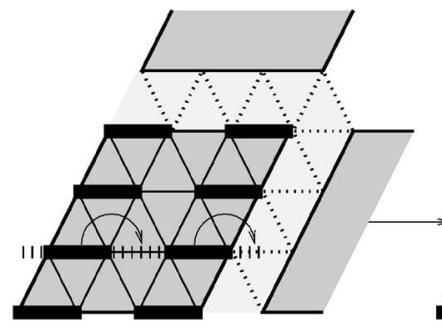

$\operatorname{TS}(0,0)$

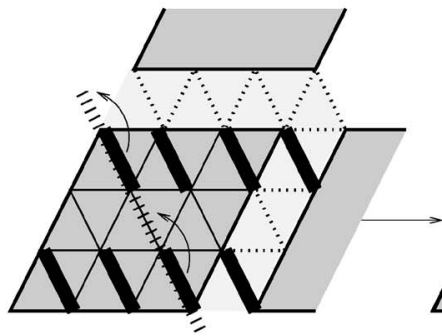

$\operatorname{TS}(0,0)$

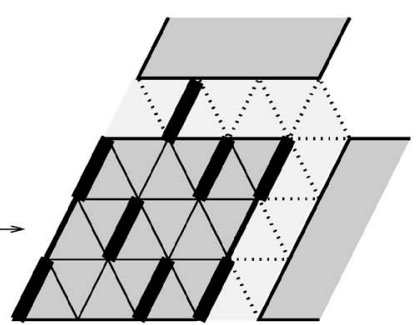

$\operatorname{TS}(1,0)$

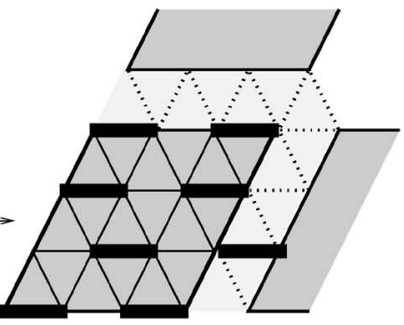

$\operatorname{TS}(0,1)$

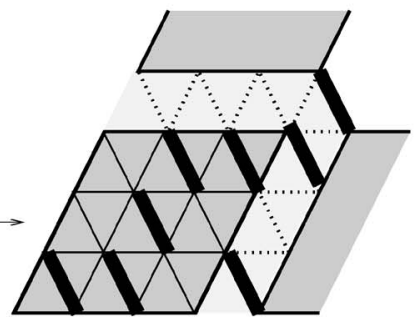

$\operatorname{TS}(1,1)$
FIG. 16. Examples of maximally flippable plaquette configurations in all sectors for $l / 2$ even. 
we could not obtain reliable evidence that it diverges, as would be the case for a continuous phase transition. In that respect, it would be very useful to implement a dynamical version of the GFMC algorithm to calculate the excitation gaps to vison and nonvison excitations inside a given topological sector (thus extending the results of Ref. 17 beyond the RK point). Work is in progress along these lines.

\section{ACKNOWLEDGMENTS}

We acknowledge very useful discussions on several aspects of this work with Grégoire Misguich and Matthias Troyer. This work was supported by the Swiss National Fund and by MaNEP. F.M. acknowledges the hospitality of SISSA, where this project started. F.B. is supported by INFM.

\section{APPENDIX A: RELATIONS BETWEEN TOPOLOGICAL SECTORS}

As stated in sec. II C and summarized in Fig. 4, the topological sectors $(0,1)$ and $(1,0)$ are always degenerate either with $(0,0)$ or $(1,1)$ depending on the cluster size. The reason for this is simply that three out of four topological sectors are related by $\pi / 3$ rotations. In order to know to which topological sectors $(0,1)$ and $(1,0)$ are connected, it is useful to keep track of the parity of the number of dimers intersecting a line in the third direction, i.e., $\mathbf{u}_{2}-\mathbf{u}_{1}$ (see Fig. 15). A rotation by $\pi / 3$ corresponds to a cyclic permutation of the parities along the three cut lines. For the sector $(0,0)$, the parity along the third line is clearly 0 if $l / 2$ is even and 1 if $l / 2$ is odd, as illustrated in Fig. 15 for type-A clusters. Thus, if $l / 2$ is even, the $(0,0)$ sector is left unchanged by a rotation, and $(0,1)$ and
$(1,0)$ are connected to $(1,1)$. Instead, if $l / 2$ is odd, the $(0,0)$ sector is connected to $(0,1)$ and $(1,0)$, and $(1,1)$ is left unchanged.

\section{APPENDIX B: EXACT DIAGONALIZATIONS}

For further reference, we list in Table II the energies obtained with exact diagonalizations for the $4 \times 4$ and the 6 $\times 6$ clusters of type A and for the 12-site cluster of type B.

\section{APPENDIX C: ERGODICITY AND MAXIMALLY FLIPPABLE PLAQUETTE CONFIGURATIONS}

If $t=0$ and $v<0$, the ground-state manifold is highly degenerate: It contains all the maximally flippable plaquette configurations. These configurations have the largest possible number of flippable plaquettes, i.e., $N / 2$. For $l / 2$ odd, they can only be found in the sectors $(0,0),(0,1)$, and $(1,0)$. For $l / 2$ even, such configurations are present in all sectors (see Fig. 16 for an example). For large negative but finite $v / t$, the degeneracy is lifted at second order in $t / v$ in favor of columnar states. ${ }^{6}$ The investigation of the ground-state properties by GFMC in this range of $v / t$ suffers from the complicated energy landscape of the phase space. The large energy barriers that separate the configurations tend to trap the GFMC simulations in metastable minima, and exponentially long simulation times would be necessary to converge to the real minimum. This situation is different from the usual behavior where the convergency to the ground state is related to the physical gap. To make sure that such problems were absent in the results presented in this paper, we have systematically compared the outcome of several simulations, starting with different pools of walkers. For each cluster size, this has defined the lowest reachable value of $v / t$.
${ }^{1}$ B. Shastry and B. Sutherland, Physica B 108, 1069 (1981).

${ }^{2}$ See, for instance, G. Misguich and C. Lhuillier, e-print cond-mat/ 0310405.

${ }^{3}$ X.-G. Wen, Phys. Rev. B 44, 2664 (1991).

${ }^{4}$ See, e.g., C. K. Majumdar and D. K. Ghosh, J. Math. Phys. 10, 1388 (1969), N. Read and S. Sachdev, Phys. Rev. Lett. 66, 1773 (1991), and Ref. 2 for recent developments.

${ }^{5}$ D. S. Rokhsar and S. A. Kivelson, Phys. Rev. Lett. 61, 2376 (1988); B. Sutherland, Phys. Rev. B 37, R3786 (1988).

${ }^{6}$ R. Moessner and S. L. Sondhi, Phys. Rev. Lett. 86, 1881 (2001).

${ }^{7}$ P. W. Anderson, Science 235, 1196 (1987).

${ }^{8}$ L. B. Ioffe, M. V. Feigel'man, A. Ioselevich, D. Ivanov, M. Troyer, and G. Blatter, Nature (London) 415, 503 (2002).

${ }^{9}$ P. Fendley, R. Moessner, and S. L. Sondhi, Phys. Rev. B 66, 214513 (2002).

${ }^{10}$ A. Ioselevich, D. A. Ivanov, and M. V. Feigel'man, Phys. Rev. B 66, 174405 (2002).

${ }^{11}$ B. Bernu, P. Lecheminant, C. Lhuillier, and L. Pierre, Phys. Rev. B 50, 10048 (1994).

${ }^{12}$ Nandini Trivedi and D. M. Ceperley, Phys. Rev. B 41, 4552 (1990).
${ }^{13}$ M. Calandra Buonaura and S. Sorella, Phys. Rev. B 57, 11446 (1998).

${ }^{14}$ The value of $\operatorname{Im}\left(\xi^{-1}\right)$ for the fermionic Green's function (3) reported in Ref. 9 is $\pi / 2-0.12 \pi$, which differs from the one in Ref. 10 due to a different gauge convention for the fermions. See footnote 15 on the sign ambiguity in the relation between the topological gaps and fermionic Green's functions.

${ }^{15}$ Note that the discussion in Ref. 10 relates the topological gap to the fermionic Green's function only up to a sign. The relative sign between the fermionic Green's function and topological gaps depends on a gauge convention for the fermions and is left undetermined in Ref. 10. In our clusters, this implies an ambiguity in a possible extra factor $(-1)^{l / 2}$. Thus the observed $(-1)^{l / 2}$ behavior of the topological gap in type B clusters is consistent with the result of Ioselevich et al. about purely real $\xi^{-1}$ in the direction $\mathbf{u}_{1}+\mathbf{u}_{2}$. Recovering the missing $(-1)^{l / 2}$ factor would require a more elaborate calculation along the lines of Ref. 10.

${ }^{16}$ R. Moessner and S. L. Sondhi, Phys. Rev. B 63, 224401 (2001).

${ }^{17}$ D. A. Ivanov, Phys. Rev. B 70, 094430 (2004). 